\title{
Risk factors of neonatal tetanus in Wenzhou, China: a case-control study
}

\author{
Zhou Zu-Mu, a Shi Hong-Ying, ${ }^{b}$ Xu Yi, ${ }^{a}$ Hu Cai-Song, ${ }^{a}$ Zhang Xiao-Ming, ${ }^{a}$ Zhao Li-Na ${ }^{a}$ and Xie Zuo-Kai ${ }^{a}$ \\ Correspondence to Zhou Zu-Mu (email: zhouzumu@126.com).
}

Introduction: Neonatal tetanus is a major cause of neonatal mortality in many developing countries and remains a major public health problem. This study aimed to determine risk factors associated with neonatal tetanus in Wenzhou, China.

Methodology: Medical records of neonatal tetanus cases from 17 hospitals over a 13-year period (2000-2012) were reviewed for potential risk factors. Controls were selected from neonates with diseases other than tetanus who were admitted to the same facility during the same period. The potential risk factors of the neonatal tetanus group were compared with the control group using univariate analysis and an unconditional logistic regression model.

Results: A total of 246 neonates with tetanus and 257 controls were included in this study. Univariate analysis showed that having untrained birth attendants, home delivery, an unsterile method of delivery and being a migrant to Wenzhou were significantly different between the two groups $(P<0.001)$. Logistic regression analysis revealed that the odds of having an untrained birth attendant, home delivery and an unsterile method of delivery were significantly higher in the tetanus group than the control group (odds ratio: 1371.0; 95\% confidence interval: 206.0, 9123.5).

Conclusion: This study identified that the main risks of neonatal tetanus in cases from Wenzhou were having an untrained birth attendant, home delivery and an unsterile method of delivery. Preventive measures directed to these risk factors may reduce the occurrence of neonatal tetanus in the studied area.

T

etanus is acquired through exposure to the spores of the bacterium Clostridium tetani, which is universally present in the environment. The organism usually prevails in dirty wounds or for neonatal tetanus, in the umbilicus following unsterile delivery. ${ }^{1,2}$ Neonatal tetanus is a major cause of neonatal mortality in many developing countries and remains a major public health problem. ${ }^{1,3,4}$ The World Health Organization (WHO) estimates that 58000 newborn infants died from tetanus in 2010. ${ }^{5}$ Although this estimate is a $93 \%$ reduction in deaths from the late $1980 \mathrm{~s},{ }^{5}$ the disease continues to occur in developing countries as well as in certain economically disadvantaged regions of China. ${ }^{6,7}$ The deaths predominantly occur in low- and middle-income countries, mostly in Asia and Africa. ${ }^{8,9}$

The incidence and mortality of neonatal tetanus differ between regions and countries and between urban and rural areas within countries. ${ }^{8,10}$ The incidence of neonatal tetanus in China has dramatically decreased from 0.2 cases per 100000 population in 2005 to 0.05 cases per 100000 population in 2012. ${ }^{11}$
Neonatal tetanus has many determinants, and many international and national efforts are aimed at eliminating neonatal tetanus. ${ }^{5,7}$ In some resourcepoor settings of the world, unsterile deliveries and poor post-natal hygiene compound the risk of neonatal tetanus. ${ }^{12-15}$ The cultural diversity of childbirth practices and cord management, untrained birth attendants, uneducated parents, poor antenatal care and lack of immunization against tetanus have also been associated with the disease. ${ }^{4,5,16,17}$ Furthermore, seasonality, geographical location, climate, prevalence of $C$. tetani and a rural agricultural population are also related to the incidence of neonatal tetanus. ${ }^{18}$

Wenzhou is a prefecture-level city in southeastern Zhejiang province, China and comprises three municipal districts and eight counties with a total land area of $11784 \mathrm{~km}^{2}$. The population of Wenzhou area is 9122 102, which includes 2842241 migrants from other areas within China; these migrants account for $31.2 \%$ of the total population. ${ }^{19}$

\footnotetext{
Department of Emergency Response, Wenzhou Center for Disease Control and Prevention, Wenzhou 325000, People's Republic of China.

Department of Preventive Medicine, Wenzhou Medical University, Wenzhou 325000, People's Republic of China.

Department of Medical Records, The Second Affiliated Hospital of Wenzhou Medical University, Wenzhou 325000, People's Republic of China.

Submitted: 23 March 2015; Published: 6 July 2015

doi: 10.5365/wpsar.2015.6.1.020
} 
Figure 1. Geographic distributions of hospitals in Wenzhou, China, 2012

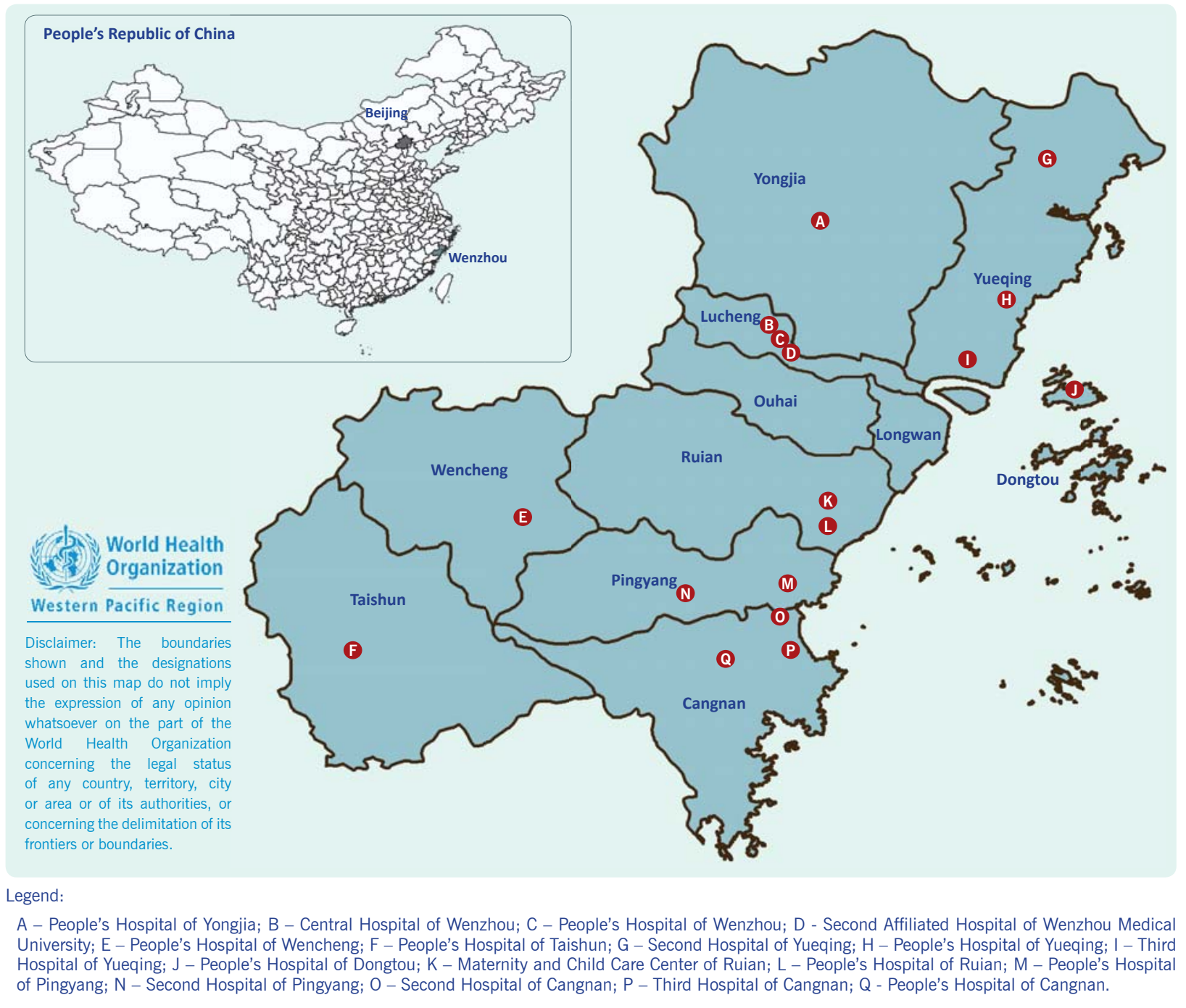

In the wake of urbanization and industrialization in

\section{METHODS}

China, domestic migration will be increasingly frequent.

There were 230 million domestic migrants in China in 2011 , accounting for $17 \%$ of the total population. ${ }^{20}$ The migrant population ratio in Wenzhou is higher than for all of China, and $87.7 \%$ of migrants in Wenzhou are people from the economically disadvantaged provinces of Guizhou and Yunnan. ${ }^{19}$ In Guizhou province, the number of cases of neonatal tetanus decreased from 276 in 2005 to 29 in 2012 and in Yunnan province from 189 cases in 2005 to 64 in 2012. These neonatal tetanus cases represent the highest number of cases at the provincial level in China. ${ }^{11}$

As the number of domestic migrants has been increasing in Wenzhou in recent years and the risk factors for neonatal tetanus are not well known in this area, this study aimed to determine the risk factors for neonatal tetanus in Wenzhou, China.

A case-control study was conducted in 17 of 24 hospitals with paediatric wards in Wenzhou, China (Figure 1). These hospitals are located in 11 counties and districts of Wenzhou. Neonatal tetanus cases diagnosed between 1 January 2000 and 31 December 2012 were identified from the medical records departments; all diagnoses were made and recorded by clinical doctors from the hospitals. The cases were verified against neonatal tetanus cases reported in the national disease surveillance information system for the same time period.

The WHO case definition for neonatal tetanus was used: a confirmed case was defined as any neonate with the normal ability to suck and cry during the first two days of life, and who between three and 28 days of age could not suck normally, or became stiff or had 
convulsions or both. ${ }^{21}$ Cases not meeting this case definition were excluded.

Controls were selected from the same medical records departments. Using either the patient registry or computer records, neonates with other diseases (such as pneumonia, congenital heart disease, gastroenteritis, neonatal jaundice, etc.) admitted to the same facility during the same time period as the cases were selected as controls.

Information about cases and controls was collected from medical records, and data on risk factors for neonatal tetanus were entered into an Excel database designed for the study. The data included age at presentation, gender, maternal age and parity, gestational age, type of inhabitants (local inhabitants, migrants from other areas in Zhejiang province or migrants from other provinces), method of delivery (sterile or unsterile), location of delivery and whether the birth attendants were trained or untrained. An unsterile delivery was defined as delivering a baby at home by untrained attendants or cord managed by a non-health care provider. Births in a hospital, health centre or clinic were categorized as births at a health facility. As three of the variables (method of delivery, place of delivery and training of birth attendants) were correlated, a new variable for overall delivery was created where category 1 was birth at a health facility with a trained attendant in a sterile environment; category 2 was either of the following - home birth, or untrained birth attendant or unsterile birth; and category 3 was a home birth with an untrained attendant in an unsterile environment. The data were entered into the database twice, and any discrepancies corrected.

Potential risk factors (including method of delivery, training of birth attendants, place of delivery, type of inhabitants, parity of the mother, neonatal gender, maternal age and neonatal weight) were compared between the cases and controls using chi-square tests or categorical data and Student $t$ test for continuous data, i.e. maternal age variable. Variables significant $(P<0.05)$ in univariate analysis were included in an unconditional logistic regression model. For all analyses, a significance level of $5 \%$ and $95 \%$ confidence interval (CI) was considered. Statistical analysis was conducted with SPSS Statistics 14.0 software (IBM, Shanghai, China).
The Wenzhou Center for Disease Control and Prevention ethical committee approved this study (No 201301).

\section{RESULTS}

A total of 246 neonatal tetanus cases and 257 controls were included. Maternal age (the mean \pm standard deviation among the case group was $27.2 \pm 4.8$ years compared with $27.4 \pm 4.8$ years for the control group, and this was not significantly different $(P=0.58)$. The gender of the neonates was also not significantly different between the two groups $(P=0.14)$.

\section{Univariate analysis}

Factors significant at the univariate level included being a migrant, having a home birth, untrained birth attendants, unsterile deliveries, increasing neonatal weight and having a multiparous mother (Table 1). For overall delivery, having one of three factors (a home birth, untrained birth attendants or unsterile delivery) had a high risk of neonatal tetanus (odds ratio [OR]: 56.0; 95\% Cl: 18.3-171.0), while having all three factors had a much higher risk of neonatal tetanus (OR: 677.5; 95\% Cl: 258.1-1778.8) (Table 1). Stratified analysis showed that the influence of neonatal gender, maternal parity, type of inhabitant, maternal age and neonatal weight on the three risk factors (home deliveries, untrained birth attendants, unsterile deliveries) was not significant.

\section{Multivariate analysis}

In the multivariate model, having one of the three birthing factors - a home birth, untrained birth attendants or unsterile delivery - and having all three remained as significant risk factors for neonatal tetanus (OR: 36.2; 95\% Cl: 5.9-221.9 and OR: 1371.0; 95\% Cl: 206.0-9123.5, respectively) (Table 2 ).

\section{DISCUSSION}

This study identified delivery by untrained attendants, home births and unsterile deliveries as risk factors for neonatal tetanus in Wenzhou city in south-eastern Zhejiang province, China, similar to that previously reported. ${ }^{12-15}$ Poverty, low maternal and paternal education, rural residence, young maternal age, cultural 


\section{Table 1. Univariate analysis of potential risk factors of neonatal tetanus, Wenzhou, China, 2000-2012}

\begin{tabular}{|c|c|c|c|c|}
\hline Characteristic* & Cases $n(\%)$ & Controls $n(\%)$ & OR (95\% Cl) & $P$-value \\
\hline Inhabitant & & & & $<0.001$ \\
\hline Wenzhou area & $142(58.2)$ & $209(81.3)$ & Reference & \\
\hline Migrants & $102(41.8)$ & $48(18.7)$ & $3.2(2.1-4.8)$ & \\
\hline Places of delivery & & & & $<0.001$ \\
\hline Health facility & $14(5.7)$ & 237 (93.3) & Reference & \\
\hline Home & $230(94.3)$ & $17(6.6)$ & $229.0(110.3-475.3)$ & \\
\hline Birth attendants & & & & $<0.001$ \\
\hline Trained & $11(4.6)$ & $241(94.5)$ & Reference & \\
\hline Untrained & $225(95.4)$ & $14(5.5)$ & $352.1(156.6-791.8)$ & \\
\hline Method of delivery & & & & $<0.001$ \\
\hline Sterile & $6(2.6)$ & 242 (94.9) & Reference & \\
\hline Unsterile & $229(97.4)$ & $13(5.1)$ & $710.5(265.6-1900.8)$ & \\
\hline Overall delivery $^{\dagger}$ & & & & $<0.001$ \\
\hline Category 1 & $7(2.9)$ & 235 (92.2) & Reference & \\
\hline Category 2 & $15(6.1)$ & $9(3.5)$ & $56.0(18.3-171.0)$ & \\
\hline Category 3 & $222(91.0)$ & $11(4.3)$ & $677.5(258.1-1778.8)$ & \\
\hline Maternal parity & & & & $<0.001$ \\
\hline Multiparous & $83(76.9)$ & $108(42.2)$ & Reference & \\
\hline Primiparous & $25(23.1)$ & $148(57.8)$ & $0.2(0.1-0.4)$ & \\
\hline Neonatal gender & & & & 0.136 \\
\hline Male & $170(74.2)$ & $175(68.1)$ & Reference & \\
\hline Female & $59(25.8)$ & $82(31.9)$ & $1.3(0.9-1.4)$ & \\
\hline Mother age (years) & & & & 0.50 \\
\hline 23 and below & $58(24.4)$ & $52(20.6)$ & Reference & \\
\hline $24-26$ & $49(20.6)$ & $69(27.3)$ & $0.6(0.4-1.1)$ & \\
\hline $27-28$ & $39(16.4)$ & $37(14.6)$ & $0.9(0.5-1.7)$ & \\
\hline $29-31$ & $49(20.6)$ & $50(19.7)$ & $0.9(0.5-1.5)$ & \\
\hline 32 and above & $43(18.0)$ & $45(17.8)$ & $0.9(0.5-1.5)$ & \\
\hline Neonatal weight (grams) & & & & $<0.001$ \\
\hline$\leq 2500$ & $15(8.0)$ & $77(32.8)$ & Reference & \\
\hline $2501-2950$ & $37(19.8)$ & $42(17.6)$ & $4.5(2.2-9.2)$ & \\
\hline $2951-3200$ & $51(27.3)$ & $37(15.5)$ & $7.1(3.5-14.2)$ & \\
\hline $3201-3500$ & $52(27.8)$ & $40(16.7)$ & $6.7(3.3-13.3)$ & \\
\hline$\geq 3501$ & $32(17.1)$ & $43(18.0)$ & $3.8(1.9-7.8)$ & \\
\hline
\end{tabular}

* Some characteristics do not add up to the total due to missing responses.

$\dagger$ Category 1 - birth at a health facility with a trained attendant in a sterile environment; Category 2 - either of the following: home birth, untrained birth attendant or unsterile birth; and Category 3 - home birth with an untrained attendant in an unsterile environment.

$\mathrm{Cl}$, confidence interval; $\mathrm{OR}$, odds ratio. 
Table 2. Logistic regression analysis of risk factors of neonatal tetanus, Wenzhou, China, 2000-2012

\begin{tabular}{|c|c|}
\hline Characteristics & OR (95\% Cl) \\
\hline \multicolumn{2}{|l|}{ Inhabitant } \\
\hline Wenzhou area & Reference \\
\hline Migrants & $3.8(0.8-17.3)$ \\
\hline \multicolumn{2}{|l|}{ Overall delivery* } \\
\hline Category 1 & Reference \\
\hline Category 2 & $36.2(5.9-221.9)$ \\
\hline Category 3 & $1371.0(206.0-9123.5)$ \\
\hline \multicolumn{2}{|l|}{ Maternal parity } \\
\hline Multiparous & Reference \\
\hline Primiparous & $0.7(0.2-3.2)$ \\
\hline \multicolumn{2}{|l|}{ Neonatal weight (grams) } \\
\hline$\leq 2500$ & Reference \\
\hline $2501-2950$ & $9.3(0.9-94.6)$ \\
\hline $2951-3200$ & $1.9(0.2-19.4)$ \\
\hline $3201-3500$ & $7.1(0.8-62.0)$ \\
\hline$\geq 3501$ & $3.7(0.4-36.8)$ \\
\hline \multicolumn{2}{|c|}{$\begin{array}{l}\text { Category } 1 \text { was birth at a health facility with a trained attendant } \\
\text { in a sterile environment; category } 2 \text { was either of the following } \\
\text { - home birth, or untrained birth attendant or unsterile birth; and } \\
\text { category } 3 \text { was a home birth with an untrained attendant in an } \\
\text { unsterile environment. }\end{array}$} \\
\hline \multicolumn{2}{|c|}{$\mathrm{Cl}$, confidence interval; and $\mathrm{OR}$, odds ratio. } \\
\hline
\end{tabular}

restrictions on access to health services for pregnant women from resource-poor regions associated with unhygienic practices, low antenatal care attendance and inadequate vaccination with tetanus toxoid have all been recognized as risk factors for neonatal tetanus. ${ }^{2,6,15,22,23}$

Untrained birth attendants often lack knowledge about sterilization and therefore use unsterile delivery appliances. The practice of cutting the cord with unsterile instruments by birth attendants is highly prevalent in the migrant population from Yunnan and Guizhou provinces; ${ }^{8}$ traditionally, birth attendants throw a bowl onto the ground and use a piece of the broken porcelain to cut the umbilical cord. ${ }^{24}$ During this investigation, this practice was recorded in the records of some pregnant migrant women. It is these unhygienic birthing practices that favour infection by $C$. tetani causing neonatal tetanus. Therefore, it is critical to target the migrant population for health promotion efforts to reduce neonatal tetanus, using interventions such as improved antenatal care, tetanus toxoid immunization of mothers, promotion of hygienic delivery, postpartum cord-care and relevant health education. . $^{8,25,26}$
There were some limitations in this study. Some known risk factors of neonatal tetanus, including the education level of the parents and immunization against tetanus were not recorded in the medical records and, hence, could not be assessed. The sample size was small, resulting in large confidence intervals. Using controls selected from hospital records also has the potential for bias as they may not represent the total population. This, as well as the location of our study and demographics of participants, suggests that the results obtained may not fully be representative of other areas or countries with different backgrounds. However, the risk factors identified in this study have all been documented before. $^{4,5,12-17}$

The present study identified having an untrained birth attendant, home birth and unsterile deliveries were risk factors for neonatal tetanus in Wenzhou, China. To eliminate neonatal tetanus in this area, targeted measures that focus on improving the skills of birthing attendants as well as promoting hospital deliveries, tetanus immunization of pregnant mothers and health education are all recommended.

\section{Conflicts of interest}

None declared.

\section{Funding}

This work was supported by a grant from the National Natural Science Foundation of China (grant no. 61373005) and the Wenzhou Science and Technology Project (grant no. Y20120006).

\section{References:}

1. World Health Organization. Tetanus vaccine. Weekly Epidemiological Record, 2006, 81:198-208. pmid: 16710950

2. Bairwa $\mathrm{M}$ et al. India is on the way forward to maternal and neonatal tetanus elimination! Human Vaccines \& Immunotherapeutics, 2012, 8:1129-1131. doi:10.4161/ hv.20262 pmid:22854674

3. Lawoyin TO. Infant and maternal deaths in rural south west Nigeria: a prospective study. African Journal of Medicine and Medical Sciences, 2007, 36:235-241. pmid:18390063

4. Oyedeji $\mathrm{OA}$ et al. Trends in neonatal and post-neonatal tetanus admissions at a Nigerian teaching hospital. The Journal of Infection in Developing Countries, 2012, 6:847-853. doi:10.3855/jidc.2105 pmid:23276738 
5. Maternal and neonatal tetanus (MNT) elimination: the initiative and challenges. Geneva, World Health Organization, 2013 (http://www.who.int/immunization/diseases/MNTE initiative/en/, accessed 21 March 2014

6. Ibinda $F$ et al. Incidence and risk factors for neonatal tetanus in admissions to Kilifi County Hospital, Kenya. PLOS ONE, 2015, 10:e0122606. doi:10.1371/journal.pone.0122606 pmid:25849440

7. Thwaites $\mathrm{CL}$, Beeching NJ, Newton CR. Maternal and neonatal tetanus. Lancet, 2015, 385:362-370. doi:10.1016/S01406736(14)60236-1 pmid:25149223

8. Roper MH, Vandelaer JH, Gasse FL. Maternal and neonatal tetanus. Lancet, 2007, 370:1947-1959. doi:10.1016/S01406736(07)61261-6 pmid:17854885

9. Lambo JA, Nagulesapillai T. Neonatal tetanus elimination in Pakistan: progress and challenges. International Journal of Infectious Diseases, 2012, 16:e833-42. doi:10.1016/j. ijid.2012.07.015 pmid:22940280

10. Datta SS et al. Three cases of neonatal tetanus in Papua New Guinea lead to development of national action plan for maternal and neonatal tetanus elimination. Western Pacific Surveillance and Response Journal, 2013, 4(2):40-43. doi:10.5365/wpsar.2013.4.1.008 pmid:24015370

11. Public health science data [In Chinese]. Beijing, Chinese Center for Disease Control and Prevention, 2004 (http://www. phsciencedata.cn/Share/ky_sjml.jsp?id=ce793729-da4c-48ebb67d-408410a59319, accessed 15 June 2015).

12. Gitta SN et al.; Centers for Disease Control and Prevention (CDC). Risk factors for neonatal tetanus-Busoga region, Uganda, 2002-2003. MMWR Morbitity and Mortality Weekly Report, 2006, 55 Suppl 1;25-30. pmid:16645579

13. Raza SA et al. A matched case-control study of risk factors for neonatal tetanus in Karachi, Pakistan. Journal of Postgraduate Medicine, 2004, 50:247-252. pmid:15623963

14. Chai F et al. Neonatal tetanus incidence in China, 1996-2001, and risk factors for neonatal tetanus, Guangxi Province, China. International Journal of Epidemiology, 2004, 33:551-557. doi:10.1093/ije/dyh073 pmid:15155708

15. Khan AA, Zahidie A, Rabbani F. Interventions to reduce neonatal mortality from neonatal tetanus in low and middle income countries-a systematic review. BMC Public Health, 2013, 13:322. doi:10.1186/1471-2458-13-322 pmid:23570611

16. Hassan B et al. A survey of neonatal tetanus at a district general hospital in north-east Nigeria. Tropical Doctor, 2011, 41:18-20. doi:10.1258/td.2010.100310 pmid:21172902

17. Dey AC, Saha L, Shahidullah M. Risk factors, morbidity and mortality of neonatal tetanus. Mymensingh Medical Journal, 2011, 20:54-58. pmid:21240163

18. Lambo JA et al. Epidemiology of neonatal tetanus in rural Pakistan. JPMA: The Journal of the Pakistan Medical Association, 2011, 61:1099-1103. pmid:22125987

19. Major figures of Wenzhou in 2010 national population census [In Chinese]. Wenzhou, Wenzhou Statistics Bureau, 2011 (http:// www. wzstats.gov.cn/info view.jsp?id0 $=$ zOh8Inkbkw\&id $1=$ zOh8l o5459\&id=zOhbddvmw $\overline{1}$, accessed 22 March 2014).

20. National Health and Family Planning Commission of the People's Republic of China. 2012 report on China's migrant population development [In Chinese]. Beijing, China population publishing house, 2012

21. WHO recommended strategies for the prevention and control of communicable diseases. Geneva, World Health Organization and the Joint United Nations Programme on HIV/AIDS, 2001 (http:// whqlibdoc.who.int/hq/2001/WHO_CDS_CPE_SMT_2001.13.pdf, accessed 15 June 2015).

22. Thind A. Determinants of tetanus toxoid immunization in pregnancy in rural Bihar. Tropical Doctor, 2005, 35:75-77. doi:10.1258/0049475054036977 pmid:15970024

23. Afridi NK et al. Coverage and factors associated with tetanus toxoid vaccination status among females of reproductive age in Peshawar. Journal of the College of Physicians and SurgeonsPakistan, 2005, 15:391-395. pmid:16197865

24. Su XH. Reform of midwife. Women of China, 2000, 12:15.

25. Darmstadt GL et al.; Lancet Neonatal Survival Steering Team. Evidence-based, cost-effective interventions: how many newborn babies can we save? Lancet, 2005, 365:977-988. doi:10.1016/ S0140-6736(05)71088-6 pmid:15767001

26. Knippenberg $\mathrm{R}$ et al.; Lancet Neonatal Survival Steering Team. Systematic scaling up of neonatal care in countries. Lancet, 2005, 365:1087-1098. doi:10.1016/S0140-6736(05)742331 pmid: 15781104 